\title{
Análisis empírico en México de estructuras de capital y su relación con las inversiones significativas (Empirical analysis of capital structures in Mexico and its relation with major investing decisions)
}

\section{Miguel Calzada Mezura}

\begin{abstract}
This study focuses on the Theories of Pecking Order and the Optimal Capital Structure (Also known as Tradeoff Theory). In relation with the theories mentioned above, previous studies described different hypothesis on financing decisions. This study, seeks to understand how companies in Mexico consider the different funding sources to invest in significant investments. The Event Study consideration is used to understand such financing decisions. The findings of this study support the Theory of Pecking Order, showing that significant investments in the shortterm are funded mainly by external sources and equity financing decisions are observed among companies that are financial constrained. This position leads to the search for different contexts in order to test the Optimal Capital Theory.
\end{abstract}

Keywords: capital structure, corporate finance, financing, investments.

JEL: G14, G31, G32, G33.

Resumen. Las Teorías del Pecking Order (Financiamiento Selectivo) y la Estructura Óptima de Capital, mencionan diferentes teoremas para las decisiones de financiamiento. El estudio busca comprender cómo las empresas en México consideran las diferentes fuentes de financiamiento para realizar inversiones significativas. La técnica de estudio de eventos es utilizada para analizar estas decisiones en empresas mexicanas. Las conclusiones del presente estudio apoyan la Teoría del Pecking Order, estableciendo que las inversiones significativas, en el corto plazo, se financian principalmente por fuentes externas y que el financiamiento de capital sólo se observa en las empresas que están limitadas financieramente. Esta postura conlleva a la búsqueda de diferentes contextos para la aplicación o rechazo de la Teoría de la Estructura Óptima de Capital.

Palabras clave: estructura de capital, financiamiento, finanzas corporativas, inversiones. 


\section{Introducción}

El entorno actual está fincado en la búsqueda constante de la mejora. En el área de finanzas dicha búsqueda ha llevado a los estudiosos a crear teorías que tratan de esclarecer lo que sucede actualmente en los mercados financieros. Como ejemplo de estos estudios, podemos mencionar la estructura de capital, en la cual se han fincado las Teorías del Pecking Order (Financiamiento Selectivo), la Teoría de la Estructura Óptima de Capital, también conocida como Tradeoff Theory (Myers, 1984), entre otras.

Esta investigación, tiene como objetivo determinar si las empresas mexicanas que cotizan en la Bolsa Mexicana de Valores, cuando enfrentan inversiones significativas, fijan su estructura de capital basados en la Teoría del Pecking Order o en la Teoría de la Estructura Óptima de Capital. Las denominadas inversiones significativas, para este estudio, están definidas como aquellas inversiones que incrementan los activos de las empresas en al menos el $20.0 \%$ con respecto del año anterior, ya sea por adquisición de nuevos activos o expansión o por adquisición de nuevos negocios.

Las decisiones de financiamiento muchas veces son relegadas por las decisiones de inversión, debido a que se tiene la idea de que se puede generar mayor riqueza con decisiones de inversión que con las llamadas decisiones de financiamiento, originando que las primeras queden en segundo término, por no ser tan valoradas por parte de la administración (Myers, 1984). El problema comienza cuando las inversiones rentables no pueden ser financiadas por falta de recursos, por tener un grado de apalancamiento demasiado elevado, causando en el futuro que no se le puede hacer frente a los compromisos financieros 0 cuando el apalancamiento crece por arriba de la industria haciendo que los inversionistas vean con riesgo a la empresa, situaciones que son conocidas como el problema de subinversión. La literatura en finanzas ha encontrado que diversos estudios muestran evidencia suficiente para inferir la presencia del Pecking Order en el comportamiento de financiamiento de las empresas (Shyam-Sunder y Myers, 1999; Wang, Meng, Huang y Huang 2011), en tanto, otros estudios muestran evidencia de la aplicación de la Teoría de la Estructura Óptima de Capital (Kayhan y Titman, 2007; Titman, y Tsyplakov, 2005).

El presente estudio confronta la Teoría del Pecking Order y la Teoría de la Estructura Óptima de Capital, a pruebas empíricas para comprender las decisiones de las empresas mexicanas cuando enfrentan decisiones de financiamiento 
para inversiones significativas. La evidencia mostrada en el presente estudio sugiere que en el corto plazo la teoría del Pecking Order explica las decisiones de financiamiento de las empresas mexicanas que cotizan en bolsa cuando estas enfrentan inversiones significativas.

\section{Metodología - Marco teórico}

De acuerdo a diversos estudios se muestran que las empresas tienden a cambiar su previa estructura de capital a una nueva estructura derivado de un financiamiento importante en inversiones significativas (Frank y Goyal, 2003; Hovakimian, Opler y Titman, 2001). Las teorías del Pecking Order (financiamiento selectivo) y la teoría de la Estructura Óptima de Capital, describen las decisiones de financiamiento en inversiones significativas de acuerdo a dos enfoques distintos (Udin, 2015). Por un lado, la teoría del Pecking Order, establece que en las empresas existe una preferencia marcada por financiar estas inversiones con recursos internos sobre recursos externos, y en dado caso que se agoten los recursos internos, la deuda será la selección preferida sobre emisión de nuevo capital social también conocido como el patrimonio (Asquith y Mullins, 1986; Baskin, 1989; Frank y Goyal, 2003; Leary y Roberts 2005; Shyam-Sunder y Myers 1999; Wang et al. 2011). De manera contraria, la teoría de una Estructura Óptima de Capital, menciona que existe un financiamiento externo óptimo y que las empresas buscarán mantener dicha estructura de capital a través del tiempo (De Angelo y Masulis, 1980; Harris y Raviv 1991; Kayhan y Titman, 2007; Stiglitz, 1988; Chirinko y Singha, 2000).

En la literatura de finanzas, se muestra que las decisiones de financiamiento y las de inversiones están relacionadas. Sin embargo, el dividir ambas decisiones en ocasiones no es sencillo, por ello, en este estudio, se analizarán únicamente las inversiones significativas debido a que estas requieren financiamiento ya sea interno o externo, logrando analizar la decisión de financiamiento que toman las empresas mexicanas cuando enfrentan un déficit para estas inversiones.

Es sabido que diferentes teorías tienen diferentes bases y razonamientos que en ocasiones se contraponen, tal como lo muestran los estudios de Shyam-Sunder y Myers (1999), Wang et al. (2011), donde encontraron evidencia estadística para sustentar la teoría de Pecking Order y en contraste, Kayhan y Titman (2007), Titman, y Tsyplakov (2005), encontraron evidencia que las empresas buscan un estándar en sus razones de endeudamiento, en otras palabras, bus- 
can la Estructura Óptima de Capital. Estas conclusiones diversas se han venido suscitando en la literatura reciente (Bradley, Jarrell y Kim 1984; Shyam-Sunder y Myers 1999; Baker, Stein y Wurgler 2002; Frank y Goyal 2003). Sin embargo, algo que se menciona en el estudio de Mayer y Sussman (2004) es que el tamaño de la empresa también tiene impacto con la decisión de financiamiento y el integrar a todas las empresas analizándolas como un todo, pudiera dar origen a un sesgo que no lleve a conclusiones correctas. Otra situación, que menciona Frank y Goyal (2003), apoyado por Udin (2015), es que las empresas rentables se comportan de manera diferente que las empresas con rentabilidad limitada 0 financieramente restringidas.

El estudio, se enfoca únicamente en las decisiones de financiamiento para inversiones significativas, dividiendo las empresas por pequeñas, medianas y grandes, en función a sus activos totales. Elsas, Flannery y Garfinkel (2006), definen que una inversión significativa es representada por un incremento en los activos totales de la empresa de un $20.0 \%$ con respecto de un año anterior. Para el presente estudio, las inversiones fueron calculadas utilizando los cambios en el balance general. La importancia de que se estudien aquellos eventos de inversiones significativas surge cuando difícilmente las empresas pueden financiar estas inversiones con una sola fuente de financiamiento y la decisión de la empresa juega un rol observable en el financiamiento. En dicha circunstancia, las empresas tendrán que decidir por su fuente de financiamiento.

La base del Pecking Order se encuentra en el estudio de Myers y Majluf (1984) donde ellos brindan las bases de la mencionada teoría. Uno de los argumentos más importantes en la teoría del Pecking Order, es la información asimétrica. La información asimétrica está definida en los estudios mencionados, como aquella situación en la que los administradores tratan de mandar señales a los inversionistas de si su empresa es "buena" o "mala". En adición, la investigación de Myers y Majluf (1984) explica que sólo los administradores saben en qué categoría se encuentra la empresa y los inversionistas interpretan las señales que mandan las empresas. Como consecuencia de la información asimétrica entre administradores e inversionistas, el valor real de la empresa o de las acciones que cotizan en la bolsa sólo es conocido por los administradores. Por ello, un administrador el cual tiene a su cargo una empresa "mala" y sabe que la cotización en el mercado de su acción esta sobrevaluada, estará tentado a emitir capital social debido a que estaría tomando ventaja de dicha sobrevaloración. En contraste, un administrador de una empresa "buena", que sabe que su 
acción esta subvaluada la emisión de nuevo capital no le será atractiva por que sabrá que estaría vendiendo porciones de la empresa a menor valor. Asimismo, los inversionistas tomarán la decisión de los administradores como información adicional a la que tienen. Como conclusión, el tema de la información asimétrica juega un rol muy importante en la decisión de emitir deuda o nuevo capital, como lo mencionan Asquith y Mullins (1986) y Baskin (1989). En adición, en una situación hipotética en la que una empresa tiene proyectos rentables y que su acción esta subvaluada, el emitir capital daría como consecuencia una transferencia de valor de los actuales accionistas a los nuevos accionistas. Como conclusión, se puede mencionar que si una empresa es "buena", la emisión de nuevo capital será su última opción por los factores mencionados anteriormente.

De manera opuesta, si la acción de la empresa está sobrevaluada, y se emite nuevo capital, se estaría transfiriendo valor de nuevos inversionistas a los inversionistas actuales. Como resultado, las empresas "malas" estarán incentivadas a emitir nuevo capital para inversiones significativas. Basado en los argumentos anteriores, los administradores de empresas "buenas" tendrán la meta de maximizar el valor de los accionistas actuales, por lo que el financiamiento para inversiones nuevas sería con recursos externos. En contraste, los administradores de las empresas "malas" buscarán explotar la sobrevaluación de su empresa y emitirán nuevo capital para financiar sus proyectos (Myers, 1993; Ross, 1977; Zhu y Wang, 2013).

Myers (1984) argumenta que el comportamiento mencionado en el ámbito financiero puede ser la razón en la determinación de la estructura de capital. Finalmente, Myers (1984) concluye que la estructura de capital es construida por la preferencia de las empresas y no buscando un óptimo de apalancamiento. Myers (1984) también establece que las nuevas inversiones primeramente se financiarán con utilidades retenidas, posteriormente deuda y finalmente con emisión de capital, este último como último recurso y con el objetivo de disminuir la probabilidad de bancarrota. De la definición de Myers (1984), en el sentido opuesto se puede inferir que las empresas "malas" prefieren recursos externos con la finalidad de explotar el sobreprecio de sus valores financieros, y buscarían la emisión de capital como principal fuente de financiamiento.

Las teorías del Pecking Order y la teoría de la Estructura Óptima de Capital, suponen una estructura de capital en la cual se maximice la riqueza de los accionistas (Shyam-Sunder y Myers, 1999). Sin embargo, de acuerdo a Myers (1984), la teoría del Pecking Order, la preferencia de financiar nuevos proyectos 
de inversión es primero utilizando recursos internos, posteriormente deuda de bajo riesgo, después la emisión de deuda con riesgo y por último nueva emisión de capital como último recurso. En tanto, la Teoría de la Estructura Óptima de Capital, busca un nivel de deuda hasta el punto máximo de los beneficios fiscales, es decir antes de que empiecen los efectos del costo de bancarrota.

Por lo tanto, la teoría del Pecking Order mostraría una preferencia por los recursos internos, en segundo término y después de haber consumido el total de los recursos internos, la deuda es la siguiente fuente de financiamiento dominante. En función a lo mencionado para mostrar la veracidad de la teoría del Pecking Order, se concluye la siguiente hipótesis:

$\mathrm{H}_{1}$ : Existe una tendencia a recurrir al financiamiento interno y posteriormente por la emisión de deuda.

En adición a la hipótesis anterior y de acuerdo con la Teoría del Pecking Order, el financiamiento con emisión de capital se deberá observar entre las empresas de baja calidad o empresas que están mayormente restringidas financieramente por ser empresas que no han sido rentables en función de sus años anteriores. Asimismo, como conclusión se presenta la siguiente hipótesis.

$\mathrm{H}_{2}$ : La emisión de capital se observa entre empresas mayormente restringidas.

La hipótesis anterior, se basa en la información asimétrica, debido a que existe una marcada intención de emitir nuevo capital entre las empresas restringidas financieramente.

\section{Metodología - Métodos y Selección de la Muestra}

La muestra para este estudio incluye 129 empresas mexicanas que reportan en Infosel Financiero y cuyo capital social o el patrimonio se negocia en la Bolsa Mexicana de Valores ("BMV"). El estudio abarca la década que comprende de 1993 a 2002, utilizando como base el Estado de Resultados y el Balance General de cada compañía. La razón principal de utilizar dicha década es la consistencia entre principios contables y evitar impactos que pudieran surgir por principios mexicanos, normas de información financiera, entre otros.

La muestra busca consistencia en los datos utilizados y excluye aquellos eventos que presentan valores atípicos en cambios en el apalancamiento de la deuda sobre los períodos $t=-1$ y $t=+1$, así como valores por arriba de $250.0 \%$ o por debajo de $-250.0 \%$. La muestra fue consistente, no obstante, se eliminaron cuatro eventos de un total de 184 . El número de observaciones se reporta en la 
Tabla 1, mostrando un número de 180 observaciones en el periodo mencionado.

Tabla 1. Distribución de Frecuencia de Inversiones Significativas de 1993 al 2002

\begin{tabular}{cc}
\hline Año & Eventos \\
\hline 1993 & 20 \\
1994 & 59 \\
1995 & 3 \\
1996 & 8 \\
1997 & 25 \\
1998 & 17 \\
1999 & 20 \\
2000 & 15 \\
2001 & 7 \\
2002 & 6 \\
\hline
\end{tabular}

Nota: 180 observaciones. Fuente: Elaboración propia.

La Tabla 2, presenta un análisis de estadística descriptiva, donde se observan las características de la muestra en relación a las inversiones significativas. En adición, dicha tabla 2 muestra tamaño, rentabilidad, crecimiento, razones de deuda y de total de pasivos sobre capital contable. Los cálculos fueron realizados basados en la muestra de las firmas que sólo tuvieron inversiones significativas.

Tabla 2. Estadística Descriptiva de las Empresas en el año de la inversión

\begin{tabular}{cccc}
\hline Variable & Media & Desv Std & Mediana \\
\hline Total activos * & 18.243 & 47.218 & 3.744 \\
Utilidad neta * & 848 & 3.859 & 117 \\
Utilidad neta (\%) & $4.6 \%$ & $8.2 \%$ & $3.1 \%$ \\
Capital * & 9.291 & 23.755 & 2.180 \\
Deuda * $_{\text {Total pasivos * }}^{*}$ & 3.802 & 11.142 & 259 \\
Pasivos (otros) * & 8.952 & 23.463 & 1.564 \\
Pasivos (otros) & 5.150 & 12.321 & 1.304 \\
razón (\%) & $28.2 \%$ & $26.1 \%$ & $34.8 \%$ \\
\hline
\end{tabular}

* Montos en millones de pesos.

Fuente: Elaboración propia. 
Las empresas mexicanas en la tabla 2 muestran desviaciones estándar importantes. Por ejemplo, los activos totales de las empresas en promedio tienen 18 mil millones de pesos. Sin embargo, la empresa más grande cuenta con 317 miles de millones de pesos, en tanto que la más pequeña contaba con tan sólo 106 millones de pesos.

\section{Construcción de Variables}

Las inversiones significativas, como muestran Elsas, Flannery y Garfinkel (2006) en su estudio, son extremadamente difíciles de financiar con una sola fuente de financiamiento. De esta manera, una ecuación de flujo de efectivo es utilizada en su estudio para determinar cómo las empresas financian sus inversiones significativas. La ecuación 1, presentada a continuación, muestra el flujo de efectivo construido para este estudio.

Inversión significativa $=$ Capital $_{\text {it }}+$ Deuda $_{\text {it }}+$ Recursos internos $_{i t}$

donde:

Capital ${ }_{t i}$ es el neto del financiamiento del capital común y preferente durante el año t.

Deuda ${ }_{t i}$ es el cambio neto de financiamiento de corto y largo plazo de deuda durante el año $t$

Recursos internos ${ }_{t i}$ es el flujo de operación, desinversiones, utilidades retenidas y cualquier otro fondo interno durante el año $t$

$i$ es el indicador de la compañía; y

$t$ es el indicador del tiempo

\section{Análisis de la Hipótesis 1}

La Tabla 3 reporta los resultados de financiamiento basados en la ecuación 1. Las razones representan una razón promedio y no el promedio de las razones de cada empresa. En otras palabras, la razón de deuda sobre inversiones (49.9 \%, t $=0$ ) es el promedio de la muestra de las empresas de nueva deuda dividida entre el promedio de las nuevas inversiones. El resto de las razones fueron calculadas de manera similar. 
En adición, para describir cómo las empresas enfrentan nuevas inversiones, este estudio presenta el flujo de efectivo de manera individual para el año del evento ( $t=0$, "año del evento") y por la suma de los años anterior y subsecuente (suma de $t=-1, t=0$ y $t=+1$ "ventana del evento"). La razón para hacer lo anterior se basa en la idea de que las empresas no necesariamente emiten nuevos valores en el año de la inversión y se busca tomar un estudio con una ventana de tiempo.

\section{Resultados para la Hipótesis 1}

La Tabla 3, muestra que principalmente las inversiones significativas son financiadas con fondos externos, principalmente deuda, conclusiones similares a Mayer y Sussman (2004) y Elsas, Flannery y Garfinkel (2006). Las empresas mexicanas con inversiones significativas emitieron deuda por aproximadamente el $50.0 \%$ de su inversión. En este caso la deuda representa la fuente más importante de financiamiento, tanto en el año del evento y la ventana del evento, lo anterior se muestra en la segunda y tercera columna respectivamente.

Tabla 3. Esquemas de Financiamiento Asociados con Inversiones Significativa

\begin{tabular}{ccc}
\hline Variable & $\begin{array}{c}\text { Evento } \\
\mathbf{t}=\mathbf{0}\end{array}$ & $\begin{array}{c}\text { Evento } \\
\mathbf{t}=(-1,+1)\end{array}$ \\
\hline Deuda / Inversiones & $49.9 \%$ & $50.3 \%$ \\
Capital /Inversiones & $23.0 \%$ & $26.0 \%$ \\
Recursos Internos /nnversiones & $27.1 \%$ & $23.7 \%$ \\
\hline
\end{tabular}

Fuente: Elaboración propia.

${ }^{*},{ }^{* *},{ }^{* * *}$ muestra que la media en la fecha del evento $\mathrm{t}=0$ es significativamente diferente de $\mathrm{t}=(-1.1)$ al $10.0 \%, 5.0 \% \mathrm{y}$ $1.0 \%$, respectivamente.

No obstante, para nuevo capital, las empresas emiten solamente el $23.0 \%$ de su inversión en el año del evento y aumentaron este financiamiento hasta un promedio de $26.0 \%$ en la ventana del evento. La segunda fuente más importante de financiamiento proviene de fuentes internas con $27.1 \%$ en el año del evento, disminuyendo a $23.7 \%$ en la ventana del estudio. Entre el año evento y la ventana del evento, la mayor diferencia muestra un incremento del $3.0 \%$ y del $3.4 \%$ para financiamiento de capital. Sin embargo, los cambios en las fuentes internas y de capital no difieren significativamente del año del evento y la ventana de eventos. 
Estos patrones de financiamiento de inversiones significativas son consistentes con la teoría del Pecking Order que indica que después de utilizar financiamiento interno, las inversiones se financian principalmente con recursos externos. La teoría también argumenta que la deuda es la fuente más importante de financiamiento cuando las empresas se enfrentan a grandes inversiones por lo que la Hipótesis 1 mencionada no se rechaza en este estudio.

A pesar de los resultados presentados hasta el momento, donde la hipótesis 1 concuerda con los estudios de Myers y Majluf (1984) y de Collins, Clement y Funke (2013), existe también evidencia que el financiamiento de capital social se puede observar entre las empresas que están mayormente restringidas financieramente hablando (Harris y Raviv, 1990; De Angelo y Masulis, 1980). La literatura reciente sugiere que este análisis debe ser analizado por el tamaño de las empresas, por lo que este estudio ahora se adentra al análisis en función del tamaño de la empresa aplicado la ecuación 1, anteriormente presentada para mostrar una solidez para la posición tomada en la hipótesis 1 y un primer análisis estadístico de la hipótesis 2.

\section{Resultados de Solidez para Hipótesis 1 y preliminares de la Hipótesis 2}

A continuación, se muestran los resultados de los Esquemas de Financiamiento para las inversiones significativas por tamaño de empresa con la intención de dar solidez a la hipótesis 1 y preliminarmente validar la hipótesis 2. La Tabla 4 muestra los resultados consistentes para tres grupos de diferente tamaño. De acuerdo con la teoría del Pecking Order, el déficit se compensa principalmente por la deuda para todas las empresas.

Las empresas de todos los tamaños utilizan la deuda como la fuente más importante de financiamiento para hacer frente a las grandes inversiones. Estos resultados son consistentes para todas las empresas en el año evento, así como en la ventana del estudio y refuerzan la conclusión de no rechazar la hipótesis 1 en función del tamaño de la empresa. En contraste, el análisis con la regresión lineal del flujo de efectivo mostrado en la ecuación 1, no es concluyente para la hipótesis 2. 
Tabla 4. Esquemas de Financiamiento Divididos por Tamaño de Empresa

\begin{tabular}{|c|c|c|}
\hline Variable & Evento $\mathrm{t}=0$ & Evento $\mathrm{t}=(-1,+1)$ \\
\hline \multicolumn{3}{|c|}{ Panel A: Empresas Pequeñas $(\mathrm{N}=60)$} \\
\hline Capital & $35.6 \%$ & $31.9 \%$ \\
\hline Deuda & $40.2 \%$ & $44.5 \%$ \\
\hline Recursos Internos & $24.2 \%$ & $23.6 \%$ \\
\hline \multicolumn{3}{|c|}{ Panel B: Empresas Medianas $(\mathrm{N}=60)$} \\
\hline Capital & $22.2 \% *$ & $32.6 \%{ }^{*}$ \\
\hline Deuda & $54.2 \%$ & $56.2 \%$ \\
\hline Recursos Internos & $23.6 \% *$ & $11.1 \% *$ \\
\hline \multicolumn{3}{|c|}{ Panel C: Empresas Grandes (N=60) } \\
\hline Capital & $12.0 \%$ & $13.6 \%$ \\
\hline Deuda & $54.8 \%$ & $49.9 \%$ \\
\hline Recursos Internos & $33.2 \%$ & $36.5 \%$ \\
\hline
\end{tabular}

Nota: la clasificación de las empresas está en función del total de activos de la muestra de acuerdo a Infosel Financiero. Fuente: Elaboración propia.

${ }^{*},{ }^{* *},{ }^{* * *}$ muestra que la media en la fecha del evento $\mathrm{t}=0$ es significativamente diferente de $\mathrm{t}=(-1.1)$ al $10.0 \%, 5.0 \%$ y $1.0 \%$, respectivamente.

Como se menciona en la hipótesis 2, existen algunas diferencias importantes entre los grupos de empresas pequeñas, medianas y grandes. En primer lugar, las empresas grandes dependen mucho más de fuentes internas que lo que el resto de las empresas lo hacen. En segundo lugar, el financiamiento a través de capital parece menos atractivo para las grandes empresas en el financiamiento de las inversiones significativas. Por el contrario, las pequeñas empresas emiten cantidades en porcentaje sustancialmente mayores de nuevo capital y menores cantidades de deuda.

Estas diferencias son consistentes con la teoría del Pecking Order y la información asimétrica, que establece que hay una marcada intención de emitir nuevo capital entre las pequeñas empresas o de aquellas que no son tan rentables, lo que en otros estudios se menciona como "malas" empresas. Por el contrario, las empresas de alto rendimiento, también conocidas como empresas "buenas" prefieren emitir deuda para evitar el problema de la subvaluación de sus acciones y utilizar el efecto de las señales al mercado para distinguirse de las compañías "malas". En resumen, los esquemas de financiamiento para las inversiones significativas muestran tres implicaciones relevantes. En primer lugar, las 
inversiones significativas se financian normalmente de múltiples fuentes, con la deuda como fuente de financiamiento dominante sobre la ventana del evento sin importar el tamaño de la empresa. En segundo lugar, las inversiones significativas son financiadas con fuentes externas. Por último, el financiamiento cuando se hacen inversiones significativas, el uso de capital común para las empresas grandes o "buenas" muestra menos influencia que para las pequeñas y medianas empresas, sin ser resultados concluyentes.

\section{Análisis adicional en los esquemas de financiamiento}

Este estudio muestra resultados concluyentes para la hipótesis 1, Sin embargo, buscando mostrar evidencia que rechace o no rechace la hipótesis 2 de la teoría del Pecking Order se tiene que analizar a mayor profundidad. Una forma de resolver o bien reducir los problemas de escala es dividir la muestra en Quintiles. En resumen, el análisis de la inversión significativa se localizará por grupo de empresas, y cada grupo se basa en el valor promedio de los activos totales para todas las empresas. Para la elaboración de los Quintiles, se tomaron las 40 inversiones más significativas en cada Quintil para ser consisten con todos los Quintiles. La Tabla 5 presenta los resultados de las 40 mayores inversiones para cada Quintil.

\section{Resultados del análisis adicional}

Los resultados mostrados en la Tabla 5, fortalecen los resultados presentados en la Tabla 4 anterior. Aun cuando el uso de la definición de las inversiones significativas considerada fue del $20.0 \%$ de incremento del total de activos de las empresas, con respecto al cierre del año anterior, los resultados muestran las mismas conclusiones. El déficit para las inversiones significativas se financia principalmente por la deuda para todas las empresas y el nuevo capital es principalmente ausente en las empresas "buenas" (Quintil IV y V).

Los resultados son consistentes para todas las empresas en el caso del año del evento y en la ventana del evento. Los resultados muestran coherencia con el estudio de Mayer y Sussman (2004). En adición, apoya la hipótesis 2 de la teoría del Pecking Order. 
Tabla 5. Esquemas de financiamiento divididos por Quintil

\begin{tabular}{|c|c|c|}
\hline Variable & Evento $\mathrm{t}=0$ & Evento $t=(-1,+1)$ \\
\hline \multicolumn{3}{|c|}{ Panel A: Empresas en el Quintil $1(\mathrm{~N}=40)$} \\
\hline Capital & $46.0 \%$ & $33.8 \%$ \\
\hline Deuda & $67.8 \%$ & $55.4 \%$ \\
\hline Recursos Internos & $-13.8 \%$ & $10.8 \%$ \\
\hline \multicolumn{3}{|c|}{ Panel B: Empresas en el Quintil $2(\mathrm{~N}=40)$} \\
\hline Capital & $25.5 \%$ & $33.1 \%$ \\
\hline Deuda & $51.2 \%$ & $51.3 \%$ \\
\hline Recursos Internos & $23.2 \%$ ** & $15.7 \%$ ** \\
\hline \multicolumn{3}{|c|}{ Panel C: Empresas en el Quintil $3(\mathrm{~N}=40)$} \\
\hline Capital & $24.3 \%{ }^{*}$ & $34.6 \%{ }^{*}$ \\
\hline Deuda & $60.2 \%$ & $46.2 \%$ \\
\hline Recursos Internos & $15.5 \%$ & $19.2 \%$ \\
\hline \multicolumn{3}{|c|}{ Panel D: Empresas en el Quintil $4(\mathrm{~N}=40)$} \\
\hline Capital & $6.8 \%$ & $25.0 \%$ \\
\hline Deuda & $49.6 \%$ & $43.3 \%$ \\
\hline Recursos Internos & $43.6 \%$ & $31.7 \%$ \\
\hline \multicolumn{3}{|c|}{ Panel E: Empresas en el Quintil $5(\mathrm{~N}=40)$} \\
\hline Capital & $9.0 \%$ & $7.5 \%$ \\
\hline Deuda & $62.0 \%$ & $56.4 \%$ \\
\hline Recursos Internos & $29.0 \%$ & $36.1 \%$ \\
\hline
\end{tabular}

Nota: El tamaño de la clasificación para los Quintiles está basado en el total de activos de la muestra.

Fuente: Elaboración propia.

${ }^{*},{ }^{* *},{ }^{* * *}$ indica que la razón media en la fecha del evento $t=0$ es significativamente diferente de $t=(-1,1)$ al $10.0 \%$, $5.0 \%$ y $1.0 \%$ respectivamente.

Por otra parte, los resultados presentados en la Tabla 5 revelan que el $62.0 \%$ de las inversiones significativas se financia con deuda y sólo el $9.0 \%$ de dichas inversiones se financia con nuevo capital considerando las empresas grandes 0 "buenas" (Quintil V). En contraste, cuando se analizan las empresas pequeñas ("malas"), el $46.0 \%$ de las inversiones significativas se financia con nuevo capital social y aproximadamente el $68.0 \%$ de la inversión se financia con deuda (Quintil I). Además, el análisis del Quintil I es el único grupo que presenta un fondo negativo de sus fuentes internas, lo que robustece la idea de que las empresas financieramente restringidas buscarán la emisión de capital. Myers y Majluf (1984). 
Los Quintiles II y III, en comparación con los Quintiles IV y V, emiten más capital pero menos que el Quintil I. En el caso de los Quintiles II y III, aproximadamente el 25.0 \% de la inversión significativa se financia con nuevo capital social. El déficit se toma principalmente por la deuda, misma situación que ocurre para los Quintiles IV y V. Hay algunas diferencias importantes entre los Quintiles, por ejemplo, el financiamiento de nuevo capital para las empresas ubicadas en los Quintiles IV y V muestran una mínima influencia cuando estas empresas enfrentan inversiones significativas.

Por el contrario, las pequeñas empresas emiten cantidades sustancialmente mayores en porcentaje de nuevo capital y menores cantidades de deuda en comparación con el resto de los Quintiles.

Por lo tanto, los resultados son consistentes con la hipótesis 2 de la teoría del Pecking Order y el modelo de Myers y Majluf (1984), que establece que hay una mayor preponderancia de la emisión de acciones entre las pequeñas empresas de baja rentabilidad o "malas".

Con el fin de mostrar que el argumento de que las emisiones de capital se encuentran entre las pequeñas empresas de baja rentabilidad, como Myers y Majluf (1984) concluyen, y que la emisión de deuda es llevada a cabo por las empresas con mayor rentabilidad.

\section{Solidez para análisis de la Hipótesis 2}

En adición a la información presentada anteriormente y las conclusiones mencionadas, la ecuación 2 y 3 muestran una regresión de las emisiones de capital y deuda, respectivamente con la variable de flujo de operación sobre activos en donde el Flujo de Operación 1, representa las empresas más pequeñas hasta llegar al Flujo de Operación 5 que representa las empresas más grandes. Con dichas ecuaciones, se incluye en el estudio una medida de rentabilidad.

Emisión de Capital $/$ Inversiones Significativas $=\beta_{1}$ Flujo Op ${ }_{1}+\beta_{2}$ Flujo $O p_{2}+\beta_{3}$ Flujo Op $p_{3}+\beta_{4}$ Flujo Op $4+\beta_{5}$ Flujo Op $p_{5}+\varepsilon_{t}$

Emisión de Deuda / Inversiones Significativas $=\beta_{1}$ Flujo Op $+\beta_{2}$ Flujo $O p_{2}+\beta_{3}$ Flujo Op $p_{3}+\beta_{4}$ Flujo Op $4+\beta_{5}$ Flujo Op $p_{5}+\varepsilon_{t}$ 


\section{Resultados de Solidez para la Hipótesis 2}

En la Tabla 6, se presentan los resultados obtenidos utilizando cinco variables, una para cada quintil y mostrando los resultados de la ecuación 2 y 3 , las cuales se integran de la siguiente manera:

Tabla 6. Regresión de Recursos Externos sobre Flujo de Operación

\begin{tabular}{ccc}
\hline Variable & $\begin{array}{c}\text { Emisiones de Capital } \\
\text { Ecuación 2 }\end{array}$ & $\begin{array}{c}\text { Emisiones de Deuda } \\
\text { Ecuación 3 }\end{array}$ \\
\hline Quintil I & $1.52^{* * *}$ & -0.38 \\
Quintil II & $(2.89)$ & $(-0.45)$ \\
& 0.39 & 0.51 \\
Quintil III & $(0.59)$ & $(0.49)$ \\
& -0.58 & -0.97 \\
Quintil IV & $(-0.89)$ & $(-0.95)$ \\
& 0.23 & 1.69 \\
Quintil V & $(0.30)$ & $(1.42)$ \\
& 0.05 & $0.77^{*}$ \\
Observaciones & $(0.17)$ & $(1.78)$ \\
Media & 40 & 40 \\
Desv. Std & 0.22 & 0.58 \\
$\mathrm{R}^{2}$ & 0.45 & 0.53 \\
\hline
\end{tabular}

Nota: Esta tabla muestra los resultados de la regresión lineal de emisión de capital sobre flujo de operación y la regresión de emisión de deuda sobre flujo de operación. La T-estadística es mostrada entre paréntesis

Fuente: Elaboración propia.

Eq. 2; Emisión de Capital / Inversiones Significativas $=\beta 1$ Flujo Op 1+ $\beta 2$ Flujo Op $2+\beta 3$ Flujo Op $3+\beta 4$ Flujo Op $4+$ $\beta 5$ Flujo Op $5+\varepsilon t$

Eq. 3; Emisión de Deuda / Inversiones Significativas $=\beta 1$ Flujo Op 1+ $\beta 2$ Flujo Op 2+ $\beta 3$ Flujo Op $3+\beta 4$ Flujo Op $4+\beta 5$ Flujo Op $5+\varepsilon t$

${ }^{*},{ }^{* \star},{ }^{* * \star}$ muestra que la es significativa al $10.0 \%, 5.0 \%$ y $1.0 \%$, respectivamente.

La Tabla 6 muestra para el Quintil V, donde la emisión de capital es la variable dependiente, el uso de nuevo capital es casi nulo para las empresas grandes o "buenas". En contraste, analizando el Quintil I, la emisión de nuevo capital es significativo, lo que sugiere que la emisión de acciones en el año de la inversión significativa es más utilizada por las empresas pequeñas de baja rentabilidad o "malas". Asimismo, en el Quintil I, la emisión de deuda no es significativa en 
comparación con el Quintil V. En adición, se puede concluir que las empresas ubicadas en el Quintil V financian principalmente las inversiones significativas con nueva deuda, concluyendo estadísticamente que la hipótesis 2 del presente estudio no se rechaza. Finalmente, la idea de que las empresas entre mayor sea su rentabilidad la atracción por nueva emisión de acciones a través de nuevo capital social es menor. Esto se puede ver como en el Quintil II, comparándolo con el Quintil IV, en la tabla 6 se muestra mayores emisiones de capital social en el Quintil II comparado con el Quintil IV y esta comparación se magnifica comparando el Quintil I y el Quintil V como ya se ha mencionado.

\section{Conclusiones}

El Pecking Order brinda una nueva perspectiva de cómo las empresas pueden tomar sus decisiones de financiamiento en términos de otras variables, dejando el beneficio fiscal como segunda instancia y contrastando con la teoría Óptima de Capital, donde en dicha teoría su mayor argumento es que la deuda cuenta con un beneficio fiscal y las empresas buscan maximizar dicho beneficio sin verse afectados por la probabilidad de la bancarrota y sus costos asociados. El Pecking Order, al igual que la teoría Óptima de Capital, busca la maximización de la riqueza de un accionista, sin embargo, como se menciona anteriormente, el beneficio fiscal está en segunda instancia debido a que toma otros factores cuantitativos, tales como la información asimétrica, oportunidades de crecimiento, disponibilidad de flujo para reparto de dividendos, problemas de subinversión, entre otros (Myers, 1984).

De acuerdo a lo mencionado anteriormente, la hipótesis 1, establece que en la teoría del Pecking Order, existe una tendencia por el financiamiento interno, posteriormente por la emisión de deuda, dejando como última instancia la emisión de acciones a través del capital social. Los resultados presentados indican que las inversiones significativas, en el corto plazo, son financiados principalmente por la emisión de nueva deuda y en menor medida por emisión de capital social. Por lo tanto, la hipótesis 1 , no se rechaza y se muestra una preferencia de financiamiento de acuerdo a la teoría del Pecking Order. En adición, como se observa en las Tablas 2 y 3 de este estudio, se muestra que principalmente las inversiones significativas son financiadas con fondos externos, principalmente con deuda, como lo sugiere la teoría del Pecking Order. Las empresas en promedio con inversiones significativas emitieron deuda por aproximadamente el $49.9 \%$ de 
su inversión, seguido de recursos internos con un $27.1 \%$ y como última instancia de financiamiento, la emisión de nuevo capital social representando el $23.0 \%$.

Asimismo, y basado en el resultado mencionado, la deuda representa la fuente más importante de financiamiento, tanto en el año del evento, como en la ventana del evento, lo anterior se muestra en la segunda y tercera columna respectivamente de la tabla 2 y 3 . Dichas tablas vuelven a indicar que la emisión de nuevo capital es el último recurso en las fuentes de financiamiento.

Continuando con el análisis final del estudio, la hipótesis 2 del presente, menciona que la deuda es el recurso de financiamiento dominante después de agotar los recursos internos y que la emisión de capital social sólo se verá en aquellas empresas que están financieramente restringidas. Para distinguir entre las empresas rentables y poco rentables, o como se menciona en el presente estudio, empresas financieramente restringidas, la presente investigación también analiza los eventos por tamaño de empresa, haciendo una división en Quintiles.

El estudio, en la tabla 6, la cual se basa en cinco grupos de empresas, se puede observar que las empresas más grandes de la muestra, a las que se les puede asumir mayor flexibilidad financiera y donde la emisión de capital social es la variable dependiente en el uso de la ecuación lineal, la emisión de nuevo capital social es casi nulo confirmando evidencia para la teoría del Pecking Order.

En contraste, analizando las empresas menos rentables o con mayores restricciones financieras, la emisión de nuevo capital social para enfrentar inversiones es significativo, lo que sugiere que la emisión de acciones en el año de la inversión es más utilizada por las empresas pequeñas de baja rentabilidad o "malas".

En adición, para las empresas restringidas financieramente, la emisión de deuda no es significativa en comparación con las empresas grandes. En resumen, se puede concluir que las empresas con mayores efectos por la información asimétrica financian principalmente las inversiones significativas con nueva deuda y el financiamiento de capital social se observa entre las firmas o empresas que, en general, es probable que sean limitadas financieramente o con una rentabilidad menor.

Con base en los resultados, se establece que en el corto plazo las empresas grandes emplean la deuda en lugar de un financiamiento con capital social y aumentan su nivel de endeudamiento de acuerdo con sus necesidades. Además, los resultados sugieren que las empresas grandes no emiten nuevas 
acciones para solventar sus necesidades de financiamiento, conclusión a la cual también el estudio de Elsas, Flannery y Garfinkel (2006) muestra los mencionados resultados.

Esta investigación presenta un modelo empírico alternativo basado en el estudio de Mayer y Sussman (2004), donde se encuentra evidencia de la presencia de la teoría del Pecking Order en las empresas en México cuando estas enfrentan inversiones superiores al $20.0 \%$ de incremento en sus activos.

Dentro de esta investigación se pueden brindar efectos que no fueron considerados en el desarrollo de la presente investigación, un ejemplo claro es que las razones que se obtuvieron para el desarrollo de la misma, fueron datos de valores en libros, pero un punto importante es qué determinación pasaría si se realiza la misma investigación con valores de mercado de los activos, con ello se sabría si los administradores toman su decisión de financiamiento con estados financieros o con información de mercado.

Un área más que se recomienda para ampliar la presente investigación es el uso de modelos de causalidad, en donde permita reflejar el efecto que tienen períodos anteriores sobre las variables explicativas dentro de la estructura de capital y si esos efectos tienen impacto en las decisiones de financiamiento de las empresas.

\section{Referencias}

Alkhazaleh, A. M. \& Almsafir, M. K. (2015). Does asymmetry of information drive banks' capital structure? Empirical evidence from Jordan. International Journal of Economics and Finance. (73), 86-97.

Asquith, P. y Mullins, D. (1986). Equity issues and offering dilution. Journal of Financial Economics, 15, 61-89.

Baker, M., Stein, J.C. y Wurgler, J. (2002). When does the market matter? Stock prices and the investment of equity-dependent firms. Quarterly Journal of Economics, 118(3), 969-1005.

Barnea, A. Haugen R. A. y Senbet, L. W. (1981). Markets imperfections, agency problems, and capital structure: A review. Financial Management, 10(3), 7-22.

Baskin, J. (1989). An empirical investigation of the pecking order hypothesis. Financial Management, 18, 26-35

Breadley, M., Jarrell, G.A. y Kim, E.H. (1984). On the existence of an optimal capital structure: Theory and evidence. Journal of Finance, 38(3), 857-878.

Chirinko, R. y Singha, A. (2000). Testing static tradeoff against pecking order models of capital structure: A critical comment. Journal of Financial Economics, 58(3), 417-425. 
Cleary, S. (1999). The relationship between firm investment and financial status. Journal of Finance, 54, 673-692.

Collins, S. O., Clement, A. A., Funke, A. R. (2013). Profitability and debt capital decision: a Reconsideration of the Pecking Order Model. International Journal of Business and Management. 8(13), 24-33.

De Angelo, H. y Masulis, R. W. (1980). Optimal capital structure under corporate and personal taxation. Journal of Finance Economic, 8, 3-29.

Elsas, R., Flannery, M. J. y Garfinkel, J. A. (2006). Major investments, firms financing decisions, and long-run performance. Manuscrito no publicado. Institute for Finance and Banking and Department of Finance. University of Florida.

Frank, M. y Goyal, V. (2003). Testing the pecking-order theory of capital structure. Journal of Financial Economics, 67, 217-248.

Harris, M. y Raviv, A. (1990). Capital structure and the informational role of debt. Journal of Finance, 37, 321-341.

Harris, M. y Raviv, A. (1991). The theory of capital structure. Journal of Finance, 46, 297-355.

Hovakimian, A., Opler T. y Titman S. (2001). The debt-equity choice. Journal of Financial and Quantitative Analysis, 36, 1-24.

Kalay, A. (1980). Signaling, information content, and the reluctance to cut dividends. Journal of Financial and Quantitative Analysis, 15, 855-869.

Kayhan, A. y Titman, S., (2007). Firms' histories and their capital structure. Journal of Financial Economics, 83, 1-32.

Leary, M. y Roberts, M. (2005). Do firms rebalance their capital structures? Journal of Finance, 6, 2575-2619.

Leland, H.E. y Pyle, D. (1977). Asymmetric information, financial structure, and financial intermediation. Journal of Finance, 32, 371-388.

Long, M. S. y Malitz, I. (1985). The investment - financing nexus: Some empirical evidence. Midland Corporate Finance Journal, 3(3), 53-59.

Mayer, C. y Sussman, O. (2004). A new test of capital structure. Manuscrito no publicado. Oxford University.

Miller, M.H. (1978). The Modigliani-Miller propositions after thirty years. Journal of Economics Perspectives, 2(4), 99-120.

Modigliani, F. y Miller, M. (1958). The cost of capital, corporate finance, and the theory of investment. American Economy Review, 48, 261-297.

Myers, S.C. (1984). The capital structure puzzle. Journal of Finance, 39(3), 575-593.

Myers, S.C. (1993). Still searching for optimal capital structure. Journal of Applied Corporate Finance, 1, 4-14.

Myers, S.C. y Majluf, N.S. (1984). Corporate financing and investment decisions when firms have information that investors do not have. Journal of Financial Economics, 13, 187-222.

Opler, T. y Titman, S. (1994). Financial distress and corporate performance. Journal of Finance, 49, 1015-1040. 
Ross, S. (1977). The determination of financial structure: The incentive signaling approach. Bell Journal of Economics, 8(1), 23-40.

Shyam-Sunder, L. y Myers, S.C., (1999). Testing static tradeoff against pecking order models of capital structure. Journal of Financial Economics, 51, 219-244

Smith, C.W. y Watts, R.L. (1992). The investment opportunity set and corporate financing, dividend and compensation policies, Journal of financial Economics, 32, 263-292.

Stiglitz, J. E. (1988). Why financial structure matters, Journal of Economics Perspectives, 2(4), 121-126.

Titman, S. y Tsyplakov, S. (2005). A dynamic model of optimal capital structure. Manuscrito no publicado, 2005.

Udin, N. (2015). Determinants of corporate capital structure: a theoretical integration and some empirical evidences. International Journal of Economics and Finance 7(7), 245-277

Wang, K., Meng, Y., Huang, F. y Huang, D. (2011). Information transparency and corporate financing decision: testing the pecking order theory. African Journal of Business Management. 5(22), 8778-8788.

Zhu, W. y Wang, Z. (2013). Equity financing constraints and corporate capital structure. China Finance Review International. 3(4), 322-339. 